\title{
Transanal Endoscopic Microsurgery
}

\author{
Byung Chun Kim \\ Department of Surgery, Hallym University Kangnam Sacred Heart Hospital, Hallym University College of Medicine, Seoul, Korea
}

\section{See Article on Page 23-27}

The use of a local excision to treat patients with early rectal cancer is increasing these days [1]. Local excisions have been commonly used because they have the benefits of decreased postoperative morbidity and mortality, good functional outcome, and avoidance of the need for a stoma [2]. However, the use of a local excision, when compared with a radical resection, such as a low anterior resection and an abdominoperineal resection, for the treatment of patients with an early rectal malignancy remains controversial because of the variable oncologic outcomes [3]. Whether a local excision is an adequate approach to a curative resection of early rectal cancer has been a subject of much debate.

A transanal excision (TAE) has been used for the treatment of patients with rectal tumors. A standard TAE is limited to tumors of less than $4 \mathrm{~cm}$ in diameter that lie within 6 to $8 \mathrm{~cm}$ of the anal verge, and it can be relatively difficult to use because it lacks precision and has poor visual quality. The TAE has benefits of having no significant learning curve or associated equipment cost. Transanal endoscopic microsurgery (TEM) was first introduced by Dr. Gerhard Buess in Germany in 1983. Now, TEM using a 40-mm operating proctoscope through which full-thickness excisions as high as $20 \mathrm{~cm}$ from the anal verge can be performed [4]. TEM is a minimally invasive technique involving an intraluminal excision of the rectal neoplasm with the use of instrumentation to maintain a stable pneumorectum, enabling a magnified view of the operating field. It also has multiple potential benefits over a TAE. TEM provides the potential benefits of an exceptionally good view of the whole rectum, precise dissection, and en bloc excisions of tumors located not only in the lower and the middle rectum, but also in the upper rectum. TEM also offers higher likelihood of

Correspondence to: Byung Chun Kim, M.D.

Department of Surgery, Hallym University Kangnam Sacred Heart Hospital, Hallym University College of Medicine, 1 Singil-ro, Yeongdeungpo-gu, Seoul 07441 , Korea

Tel: +82-2-829-5130, Fax: +82-2-849-4469, E-mail: bckimgs@hallym.or.kr

(c) 2017 The Korean Society of Coloproctology

This is an open-access article distributed under the terms of the Creative Commons Attribution NonCommercial License (http://creativecommons.org/licenses/by-nc/4.0) which permits unrestricted noncommercial use, distribution, and reproduction in any medium, provided the original work is properly cited. achieving clear resection margins, less specimen fragmentation, and lower recurrence rates, but it is associated with a steep learning curve and requires expensive equipment.

TEM has important roles in curative resections of early rectal adenocarcinomas (T1), as well as benign and carcinoid tumors, in histopathologic staging, and in palliative resections of advanced adenocarcinomas in patients medically unfit or unwilling to undergo a radical resection [5]. Some patients show a deterioration of the rectal continence function postoperatively because of the insertion of the 40-mm operating proctoscope. O'Neill et al. [6] suggested the following algorithm for the treatment of patients with rectal tumors by using TEM: T1N0 without adverse histologic features requires TEM alone; T1N0 with adverse features identified on final pathology requires TEM with postoperative chemoradiation (vs. radical resection); T2N0 requires neoadjuvant chemoradiation followed by TEM; T3 or N1 to N3 requires TEM, but only for medically unfit patients or patients who refuse radical surgery. Chemoradiotherapy remains an integral component of the multimodal treatment plan for the patients. Individuals with proximal rectal cancer, regardless of stage, often do well with a radical resection, so TEM is less likely to be used.

Since 2009, transanal minimally invasive surgery (TAMIS) has been commonly used because it allows local excisions of rectal tumors to be performed through an anal port by using standard laparoscopic instruments. TAMIS may improve outcomes because of its being a meticulous surgical technique with an enhanced field of vision, infrequent specimen fragmentation, and lower positive margin rates [7]. Robotic-assisted TAMIS is feasible and safe for the local excisions of rectal tumors because of its allowing an increased field of vision and increased control of the robotic arm [8]. TAMIS provides potential advantages over TEM, but further comparative studies are needed to evaluate the advantages.

The study that is the subject of this editorial enrolled $130 \mathrm{pa}-$ tients with rectal adenomas and early rectal cancer over a period of 6 years. The average tumor size was $2.8 \pm 1.5 \mathrm{~cm}$ (range, 0.5 to $8.3 \mathrm{~cm}$ ). One hundred two benign and 28 malignant tumors were removed. Of the latter, 23 were pT1 cancers and 5 were pT2 cancers. Two patients with pT2 cancer underwent adjuvant chemotherapy, 1 patient underwent an abdominoperineal resection, 1 patient refused further treatment, and one was lost to follow up. 
No intraoperative complications were reported, but in seven cases, postoperative complications were observed: urinary retention (4 cases), postoperative hemorrhage (2 cases), and wound dehiscence (1 case). All complications were treated conservatively. The mean postoperative hospital stay was 2.3 days. Two ( 2 of 130 , $1.5 \%$ ) local recurrences ( 1 in the benign-tumor group and 1 in malignant-tumor group) were observed. The authors of the study concluded that TEM demonstrated low complication and recurrence rates and that the technique should be recommended for the treatment of patients with a rectal adenoma and for the treatment of patients with early rectal cancer who exhibit good prognostic factors for local excision. They concluded that TEM is an effective and safe method for the treatment of patients with rectal tumors [9].

TEM is safe and feasible for local excisions of benign and malignant rectal tumors in selected patients. Obviously, further safety and efficacy evaluations of TEM are needed to assess its feasibility and expected benefits compared with other methods. Further studies with randomized trials are necessary to evaluate its oncological outcomes.

\section{CONFLICT OF INTEREST}

No potential conflict of interest relevant to this article was reported.

\section{REFERENCES}

1. You YN, Baxter NN, Stewart A, Nelson H. Is the increasing rate of local excision for stage I rectal cancer in the United States justi- fied?: a nationwide cohort study from the National Cancer Database. Ann Surg 2007;245:726-33.

2. Lev-Chelouche D, Margel D, Goldman G, Rabau MJ. Transanal endoscopic microsurgery: experience with 75 rectal neoplasms. Dis Colon Rectum 2000;43:662-7.

3. Kidane B, Chadi SA, Kanters S, Colquhoun PH, Ott MC. Local resection compared with radical resection in the treatment of T1N0M0 rectal adenocarcinoma: a systematic review and metaanalysis. Dis Colon Rectum 2015;58:122-40.

4. Buess G, Theiss R, Hutterer F, Pichlmaier H, Pelz C, Holfeld T, et al. Transanal endoscopic surgery of the rectum - testing a new method in animal experiments. Leber Magen Darm 1983;13:737.

5. Palma P, Horisberger K, Joos A, Rothenhoefer S, Willeke F, Post S. Local excision of early rectal cancer: is transanal endoscopic microsurgery an alternative to radical surgery? Rev Esp Enferm Dig 2009;101:172-8.

6. O’Neill CH, Platz J, Moore JS, Callas PW, Cataldo PA. Transanal endoscopic microsurgery for early rectal cancer: a single-center experience. Dis Colon Rectum 2017;60:152-60.

7. Martin-Perez B, Andrade-Ribeiro GD, Hunter L, Atallah S. A systematic review of transanal minimally invasive surgery (TAMIS) from 2010 to 2013. Tech Coloproctol 2014;18:775-88.

8. Hompes R, Rauh SM, Ris F, Tuynman JB, Mortensen NJ. Robotic transanal minimally invasive surgery for local excision of rectal neoplasms. Br J Surg 2014;101:578-81.

9. Dulskas A, Kilius A, Petrulis K, Samalavicius NE. Transanal endoscopic microsurgery for patients with rectal tumors: a single institution's experience. Ann Coloproctol 2017;33:23-7. 